\title{
Models of Environmental Impact Assessment of Emerging Technologies from Chemical and Cement Industry ${ }^{\dagger}$
}

\author{
Radu Claudiu Fierascu 1, Irina Fierascu ${ }^{1, *}$ and Adriana Moanta ${ }^{2}$ \\ 1 National Institute for Research \& Development in Chemistry and Petrochemistry -ICECHIM Bucharest, \\ 202 Spl. Independentei, 060021 Bucharest, Romania; radu_claudiu_fierascu@yahoo.com (R.C.F.) \\ 2 CEPROCIM SA, 6 Preciziei Str., Sector 6, 062203 Bucharest, Romania; moantaa@yahoo.com \\ * Correspondence: dumitriu.irina@yahoo.com \\ + Presented at the 15th International Symposium "Priorities of Chemistry for a Sustainable Development" \\ PRIOCHEM, Bucharest, Romania, 30th October-1st November 2019.
}

Published: 14 October 2019

Keywords: chemical industry; cement industry; greenhouse gases

Industry is a major source of global greenhouse gas emissions. The global industrial sector accounts for approximately $47 \%$ of energy-related carbon dioxide emissions, and significant quantities of additional greenhouse gases (GESs) are released as industrial process gases, such as $\mathrm{CH}_{4}$, $\mathrm{N}_{2} \mathrm{O}$, and fluorinated gases (hydrofluorocarbons-HFC, perfluorocarbons-PFC, and Sulphur hexafluoride - SF6), with the latter emissions being concentrated in the chemical industry [1]. Additionally, the cement industry is one of the major contributors for greenhouse gas (GHG) emissions, specifically $\mathrm{CO}_{2}$ emissions [2]. Regarding $\mathrm{CO}_{2}$ emissions, the global emissions of $\mathrm{CO}_{2}$ reached approximately 28.3 gigatons (Gt) in 2005, of which the cement industry generated approximately $1.8 \mathrm{Gt} \mathrm{CO}_{2}$, indicating that the cement industry contributed approximately $6 \%$ of the total global $\mathrm{CO}_{2}$ emissions [2]. In the EU-28, $\mathrm{CO}_{2}$ accounted for over $94 \%$ of $\mathrm{CO}_{2}$-equivalent GES emissions in the industrial sector, in 2012 [3].

In this context, models of environmental impact assessment of emerging technologies are critical to promote sustainable chemical production, and can provide policy makers with useful insights for the future. Increasing demand for energy and more severe environmental problems is a consequence of the globalization of industry and the increasing population. In this context, development of emerging technologies for different industries is necessary for a "cleaner future" and a decrease in energy consumption.

For models of environmental impact assessment, many parameters must be taken in consideration. Starting from the stage of RD\&D (Research, Development, and Deployment) to the final product manufacturing, evaluations must be performed in order to fulfill the greenhouse gas emission (GES) reduction goals.

In this review paper, the advantages and drawbacks for some models of environmental impact assessment are briefly described, which can be used in the technologies emerging from different industries.

Life Cycle Assessment is the most commonly used approach for evaluating both conventional and emerging technologies in the chemical industry, but sometimes can present major drawbacks due to variations in parameters, when the laboratory model is scaled to the pilot model and further to industry [4]. In this case, the pass from one scale to another is the subject of the mathematical or computing simulations [5]. Emerging technologies are also evaluated through the economic inputoutput analysis, offering an average value of all products included in one sector [6]. Other models developed by different authors like Fault Tree Analysis, Failure Mode Effect Analysis, and Hazard 
and Operability Study could also be considered [7]. These methods could be used as part of the process design procedure, especially in operation phases but they can only approach single aspects of the chemical processes.

In conclusion, the study found that the use of these models can offer the possibility to improve the processes, in a given timeframe, in order to reduce environmental footprints. Additionally, a decrease of different costs can be performed, leading to an enhancement in the decision-making related to future developments in emerging technologies.

Acknowledgments: The authors gratefully acknowledge the financial support obtained from the Sectorial Project, Contract No. 4PS/2019, from the Sectorial Program-Romanian Ministry of Research and Innovation.

\section{References}

1. OECD. Available online: https://stats.oecd.org/glossary/detail.asp?ID=1343 (accessed on 16 September 2019)

2. Zhang, J.; Liu, G.; Chen, B.; Song, D.; Qi, J.; Liu, X. Analysis of $\mathrm{CO}_{2}$ Emission for the cement manufacturing with alternative raw materials: A LCA-based framework. Energ. Proc. 2014, 61, 2541-2545.

3. European Environment Agency (EEA). Available online: https://www.eea.europa.eu/publications/approximated-eu-ghg-inventory-proxy (accessed on 16 September 2019).

4. Yao, Y.; Masanet, E. Life-cycle modeling framework for generating energy and greenhouse gas emissions inventory of emerging technologies in the chemical industry. J. Clean. Prod. 2018, 172, 768-777.

5. Morais, S.; Mata, T.M.; Martins, A.A.; Pinto, G.A.; Costa, C.A.V. Simulation and life cycle assessment of process design alternatives for biodiesel production from waste vegetable oils. J. Clean. Prod. 2010, 18, 12511259.

6. Yang, Y.; Ingwersen, W.W.; Hawkins, T.R.; Srocka, M.; Meyer, D.E. USEEIO: a new and transparent United States environmentally-extended input-output model. J. Clean. Prod. 2017, 158, 308-318.

7. Sugiyama, H.; Fischer, U.; Hungerbühler, K.; Hirao, M. Decision framework for chemical process design including different stages of environmental, health, and safety assessment. AIChE J. 2008, 54, 1037-1053. 